\section{Mitogenic neurotransmitters}

\section{Michael R. Hanley}

Despite significant progress in the description of vertebrate neural cell lineages and differentiation, little is known about how the extensive proliferation of early brain-precursor cells is coordinated, or how non-neural cells are stimulated to divide during development or following injury. The question of neural cell mitogens has focused on the actions of known growth factors, but the report of Capon and colleagues on page 146 of this issue ${ }^{1}$ calls attention to a possibility that is gaining support; that neurotransmitters themselves have the potential to act as regulators of nerve cell growth and division.

Using both transfected Chinese hamster ovary cells and various cell lines expressing identified muscarinic acetylcholine receptor subtypes, the authors note that there is a strict correspondence between the occurrence of specific muscarinic receptors and the relative ability of the stable acetylcholine analogue carbachol to drive DNA synthesis. Specifically, two muscarinic receptor subtypes

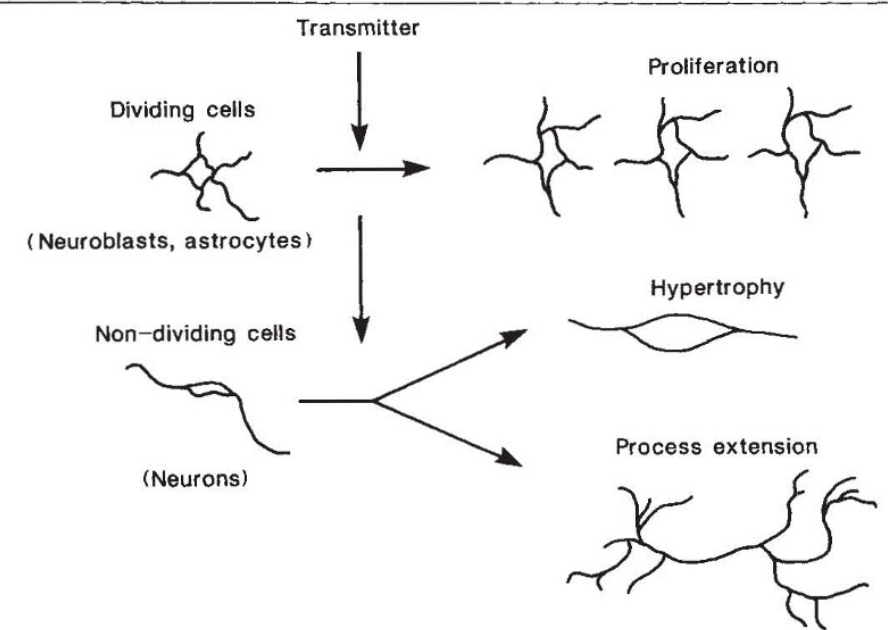

Possible neurotransmitter receptor-driven growth processes. to different signalling pathways, including the negative regulation of adenyl cyclase through the GTP-binding protein $G_{i}$ (ref. $5)$, can drive the common output event of cell proliferation. Indeed, it could well turn out that all the main second-messenger pathways regulated by G-proteincoupled receptors are conditionally mitogenic, albeit to varying extents.

Against this background, it is perhaps not suprising that two neural receptors, control (see figure). The neuronal counterpart of a hypertrophic response would not be the induction of increased somatic volume, but rather would be the stimulation of increased cytoplasmic surface area by enhancing process formation and branching. This type of 'trophic' activity is not normally considered to be within the repertoire of neurotransmitter action, but the notion that trophic forms of growth responses lie on one end of a continuum which has cell division at the other implies an appealing genetic unity to all forms of neural growth.

Neurotransmitter stimulation of proliferation must also be dependent on some other cellular events which help to define the permissive 'context'. In mas-transfected neural cells ${ }^{4}$, angiotensin acts as a direct mitogen but bradykinin does not, even though both receptors are coupled to inositol-lipid hydrolysis. There must therefore be some aspect of the angiotensin-mediated activation of these cells which is essential for a mitogenic response.

One candidate for an essential element in mitogenic action which is being widely examined is the newly defined lipid kinase called phosphatidylinositol 3-kinase or type I coupled to inositol lipid metabolism, M1 and M4, can elicit a clearcut mitogenic response, whereas two others, M2 and M3, which are coupled to negative regulation of adenylate cyclase, are relatively inefficient in eliciting DNA synthesis. (The nomenclature in this field is confusing - see Eric Barnard's News and Views article ${ }^{2}$. The receptors designated here ${ }^{1}$ M3 and M4 are referred to by many as M4 and M3, respectively.).

Capon and colleagues extend these observations to a physiologically more relevant preparation - enriched primary cultures of astrocytes from the developing brain - and find that subpopulations of astrocytes show a mitogenic response to carbachol. Thus they suggest that acetylcholine, in addition to its transmitter function, may act as a neuron-to-glial cell signal in triggering cell division. These results are part of a growing consensus that not just growth factors but a large variety of chemical messengers are able to drive DNA synthesis in the mature and the developing nervous system.

It now seems that several neural receptors coupled to phospholipase $\mathrm{C}$ hydrolysis of inositol lipids have mitogenic potential, including the cloned $5-\mathrm{HT}_{\mathrm{lc}}$ (ref. 3) and mas oncogene/angiotensin receptors ${ }^{4}$. All the muscarinic receptors so far identified can drive DNA synthesis to varying extents, implying that receptors coupled mas and the 5-HT ${ }_{i c}$ receptor, can induce a malignant state of uncontrolled growth, leading to tumours when expressed in NIH-3T3 cells ${ }^{4,6}$ - the standard indicator for oncogene identification. Thus, some neurotransmitter receptors may not only have the unexpected characteristic of mitogenic control, but also can be properly considered 'proto-oncogenes'. There is a need to assess the entire family of cloned G-protein-coupled receptors, which share the seven-hydrophobic domain pattern in amino-acid sequence ${ }^{7}$ for transforming or tumorigenic activity in susceptible cells.

The action of mitogenic neurotransmitter receptors is most readily understood in the context of early nerve cell precursors, which are still actively dividing, or in the context of non-neuronal cells such as astrocytes, which maintain a proliferative capacity in adulthood. The possibility that mitogenic neurotransmitter receptors act in novel ways on post-mitotic neurons should also be considered, however.

In cardiac myocytes, another nondividing cell population, many of the genetic responses associated with cell proliferation are maintained in receptorstimulated hypertrophy, where a cell does not divide but gains cytoplasmic mass ${ }^{8}$. neuroblasts is exclusively linked to proliferation, may therefore be retained in mature neurons for other forms of growth The genetic cascade, which in immature
PI kinase, which makes an unusual inositol phospholipid, phosphatidylinositol 3-phosphate'. The reason for the intense interest in this enzyme is that it seems to associate directly with the mitogenic platelet-derived growth factor receptor through a domain inserted in the tyrosine kinase, and the deletion of this domain leads to an abolition of mitogenic responses through the receptor ${ }^{10}$. Other evidence suggests that type I PI kinase also forms active complexes with the viral-transforming gene polyoma middle-T antigen ${ }^{11}$. Although it is not at all clear how this enzyme acts to define the mitogenic context, it provides a provocative candidate 'mitogenic switch' which might act in nerve cells, as in other cell populations, to specify whether biochemical transduction of a neurotransmitter signal leads to a specific growth response.

Michael R. Hanley is in the MRC Molecular Neurobiology Unit, University of Cambridge Medical School, Cambridge CB2 2QH, UK.

\footnotetext{
1. Ashkenazi, A., Ramachandran, J. \& Capon, D.J. Nature 340, 146-150 (1989).

2. Barnard, E. Nature 335, 301 (1988)

3. Julius, D. et al. Science 244, 1057-1062 (1989)

4. Jackson, T.R. et al. Nature 335, 437-440 (1988).

5. Seuwen, K. et al. Nature 335, 254-256 (1988).

6. Young, D. et al. Cell 45, 711-719 (1986).

7. DohIman, H.G. et al. Biochemistry 26, 2657 (1987)

8. Simpson, P.C. A. Rev. Physiol. 51, 189-202 (1988).

9. Whitman, M. et al. Nature 332, 644-646 (1988)

10. Coughlin, S.R. et al. Science 243, 1191-1194 (1989).

11. Courtneidge, S.A. \& Heber, A. Cell 50, 1031-1037 (1987)
} 